\title{
An Investigation of DC Characteristics in Multifinger Optically Illuminated MOSFET
}

\author{
Prerana Jain \\ Ph D student \\ SKVM's NMIMS, Vile Parle(W) \\ Mumbai, India
}

\author{
B.K. Mishra \\ Principal \\ TCET, Kandivali (E) \\ Mumbai, India
}

\begin{abstract}
This paper presents drain current for a long channel MOSFET and a multifinger MOSFET structure used at RF. The drain current and the transconductance are investigated for all regions of MOSFET operation under dark condition and under illumination. The MOSFET under illumination indicates its potential for analog and mixed signal applications at RF.
\end{abstract}

\section{General Terms}

Modeling, Optoelectronics, Simulation

\section{Keywords}

DC, Characteristics, Current Modeling, MOSFET, Multifinger, Optoelectronics, Transconductance

\section{INTRODUCTION}

CMOS is the most established technology due to significant advantages over all other existing technologies. In technology larger integration and higher operating speeds are the essentials. With advancement in technology, Silicon MOSFET is found to be very attractive for operation at RF. The circuitry at RF consists primarily of amplifiers, filters, mixers and oscillators. The basic step for designing the circuit is choice of appropriate biasing point. In today's technology, the trend is to design circuits operating at low supply voltage to achieve low power consumption. Due to this, limited voltage budget is associated with transistor biasing. [1] One of the ways is optoelectronic integration by illuminating the device with intensity modulated wave with energy greater than bandgap energy. Optoelectronic and RF CMOS integration offers all the benefits like immunity to electromagnetic interference, better reliability etc [2]. It also gives an additional independent control port along with all the advantages of optoelectronics. This also renders control over operating region of the device which is crucial in submicron dimension especially operating at high frequency.

For the circuit designing at RF, it is essential to understand physical device operation and deal with device characteristics and models. The device model has equivalent circuit consisting of resistances, capacitances and voltage controlled current sources. The MOSFET is a nonlinear device and the characteristics exhibit strong dependence on bias. For a MOSFET at RF though $\mathrm{Y}$ and $\mathrm{S}$ parameters are of prime importance, a lot of parameters are extracted from the DC characteristics. They represent components in the large and small signal model used at low, medium and high frequency operation. Analog and RF circuit design always begins with the knowledge of DC characterization [3]. Thus DC characteristics are equally significant in device modelling and provide the first step towards setting quiescent point of the device in the circuit.
Comparative I-V characterization of a submicron MOSFET using several models has been made in [4]. In this paper detailed analysis of drain current, conductances and transconductance has been has been made for a long channel MOSFET and multifinger MOSFET structure suitable for RF operation. Analysis has been done in all regions of operation with bias dependence for all the terminal voltage variations in dark condition and with varying optical power. Content organization of the paper is as given follows.

Section-II presents theory for MOSFET current calculations and transconductances, section-III deals with calculation of photo voltage and DC parameters under illumination. Section IV covers the results and discussion that arise out of the simulation, section- $\mathrm{V}$ deals with the major conclusions drawn from the investigation and finally the references. The optically controlled device is expected to provide an aid for device design, sizing and emerge as the prospective device for RF applications It also indicates potential for integration as optically controlled MMIC, OEICs and in ASIC .

\section{MOSFET CHARACTERISTICS}

The structure under consideration is an optically illuminated $\mathrm{N}$ - MOSFET. The N-MOSFET device is a multifinger device with $\mathrm{Nf}=10, \mathrm{Wf}=12 \mu \mathrm{m}$ and $\mathrm{Lf}=0.36 \mu \mathrm{m}$. Wf and $\mathrm{Lf}$ are effective width and length of single finger. $\mathrm{Nf}$ is the number of fingers. The device in Figure -1 represents a single finger of the device.

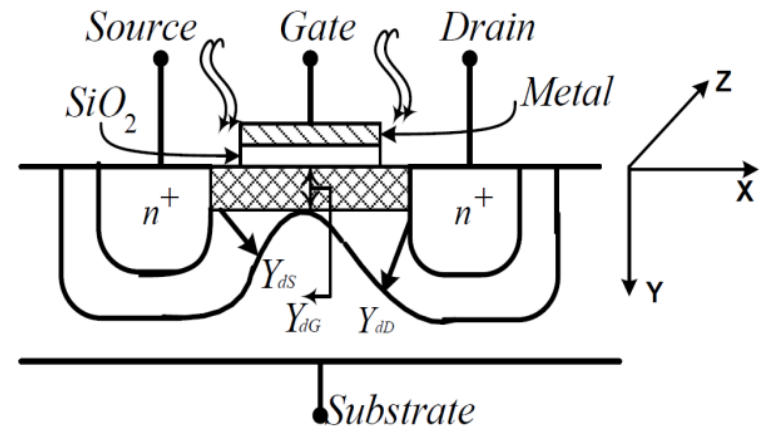

Figure 1: Schematic of single finger of MOSFET under optical radiation

When the device is to operate at RF, the MOSFETs require large width $\mathrm{W}$ to handle large power. Increasing $\mathrm{W}$ also increases the areas and perimeters of the drain and source diffusions, thereby increasing the capacitances $C_{d b}$ and $C_{s b}$ in these transistors. Exceedingly large $\mathrm{W}$ also increases the distributed effects along the gate, with a reduction in effective transistor performance. To optimize the performance, multifinger MOSFETs are used which reduce parasitic capacitances and effectively lower gate resistance $R g$ by 
factor of $1 / N_{f}$. This multifinger structure also maintains current handling capability and the transconductance of the MOSFET [5]. A general expression for the drain current that includes drift and diffusion mechanism is given by as in (1).

$I_{D}=-\mu_{e f f} \frac{W}{L} \int_{Q_{i s}}^{Q_{i}} Q_{i} \frac{\partial V_{C B}}{\partial Q_{i}} d Q_{i}$

$\mathrm{I}_{\mathrm{D}}$ is static drain current flowing into the drain terminal.

$\mathrm{V}_{\mathrm{CB}}$ is the channel voltage that varies monotonically from $\mathrm{V}_{\mathrm{S}}$ at $\mathrm{x}=0$ (source end) to $\mathrm{V}_{\mathrm{D}}$ at $\mathrm{x}=\mathrm{L}$ (drain end).

$\mu_{\text {eff }}$ Effective mobility including the effects of the vertical and longitudinal fields

$\mathrm{Q}_{\mathrm{i}}$ Inversion mobile charge density

$\mathrm{Q}_{\mathrm{iS}}$ Inversion mobile charge density at the source

$\mathrm{Q}_{\mathrm{iD}}$ Inversion mobile charge density at the drain

W Channel width, L Channel length.

A charge based approach is used as in [6]. It first calculates the dependence of the mobile inversion charge density $\mathrm{Q}_{\mathrm{i}}$ on the voltages applied to the transistor. Then, it relies on $\mathrm{Q}_{\mathrm{i}}$, and on its particular values $\mathrm{Q}_{\mathrm{is}}$ and $\mathrm{Q}_{\mathrm{iD}}$ at the source and drain ends of the channel, to calculate the drain current and transconductances. The drain current is calculated as in [7] by varying gate voltage $\left(\mathrm{V}_{\mathrm{G}}\right)$, drain voltage $\left(\mathrm{V}_{\mathrm{D}}\right)$, source voltage $\left(\mathrm{V}_{\mathrm{S}}\right)$, and substrate voltage $\left(\mathrm{V}_{\mathrm{B}}\right)$. The external resistances (for gate, source and drain) play a dominant role in the behavior of the MOSFET at RF and have been considered by modeling these as external resistances. These are calculated as in [8]. The magnitude of drain current decides region of the device operation, which in turn decides whether the MOSFET will work as switch or amplifier. The region of operation of the device depends on the applied voltages. The variation in these regions is not abrupt but is a smooth transition. The submicron devices have modified the conventional operating regions in the sense that amplifiers have shifted to moderate inversion region as against conventional long devices operated in strong inversion to get advantage of speed and power.

The drain current which depends on applied voltage bias is one the biasing point of the devices in the circuit. Another most important dc parameter is the transconductance which determines gain of the circuit. A Small-signal dc equivalent circuit is shown in Figure 2.

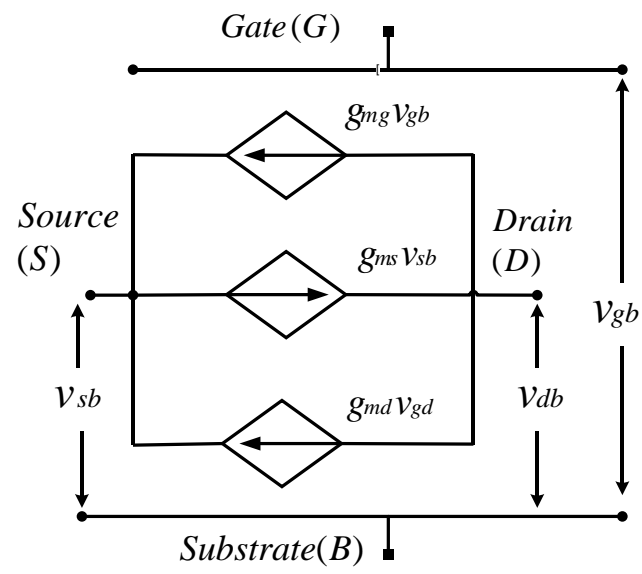

Figure 2: Small-signal dc equivalent circuit.
The transconductances are components of small-signal models of MOSFET which are valid at low, medium and high frequency. The transconductances together with the capacitances determine the transit frequency $f_{t}$ or the speed of the device, the thermal noise, and indirectly the current consumption [9]. Since the MOS transistor is a four-terminal device, it is controlled by three independent voltages, namely $\mathrm{V}_{\mathrm{G}}, \mathrm{V}_{\mathrm{S}}$, and $\mathrm{V}_{\mathrm{D}}$. A transconductance value can therefore be defined for each of these controls. The small signal parameters with bulk as the reference can be easily related to the parameters with source as the reference as shown below [10]. Gate transconductance is

$g_{m g}=g_{m}=\left[\frac{\partial I_{D S}}{\partial V_{G B}}\right] V_{S B}, V_{D B}=$ const

Drain transconductance is

$g_{m d}=g_{d s}=\left[\frac{\partial I_{D S}}{\partial V_{D B}}\right] V_{G B}, V_{D B}=$ const

Source transconductance is

$g_{m s}=\left[\frac{\partial I_{D S}}{\partial V_{S B}}\right] V_{G B}, V_{D B}=$ const

The small signal current to voltage ratio $g d s$ is known as the output conductance, as it gives the change in current flowing from drain to source for change in drain-to-source voltage. The maximum gate transconductance for a given transistor dimension (W/L) is an important parameter in analog design, and can be expressed in terms of the drain current as and also is a parameter to decide the size of the device.

\section{MOSFET UNDER ILLUMINATION}

MOSFET under illumination is a modified MIS device with is $\mathrm{Si}-\mathrm{SiO}_{2}$ interface and the device is referred to as optically gated OG-MOSFET (OG-MOSFET). Optical radiation is perpendicular to the surface and the wavelength of radiation is higher than that of silicon bandgap energy and is considered to be of $830 \mathrm{~nm}$ for simulation purpose. Optical radiation is assumed to be incident in the non- metallic gate region which will be absorbed in the depletion region.

Illumination with source $\mathrm{h} v>\mathrm{Eg}$, causes the effective potential of gate to change as photo voltage develops at the MOS junction. This also causes change in channel conductivity due to increase in mobile carriers the and hence in total charge in channel. The Qtotal is charge under illumination for the MOSFET which includes the charge in inversion and additional charge due to illumination [5]. The total charge in channel in case of optical illumination is given by

$Q_{\text {total }}=Q_{I}+Q_{\text {illumination }}$

$Q I$ is charge in the channel due to mobile carriers ,

$Q_{\text {illumination }}$ is induced charge due to illumination.

The charge due to illumination is because of generated carriers in depletion and neutral region of the channel which contributes to total channel charge. The $Q I$ and $Q_{\text {illumination }}$ are calculated as per [11,12] with appropriate boundary conditions. The total drain source current under optical radiation is obtained as

$I_{d s}=\frac{\mu \mathrm{Z}}{\mathrm{L}} \int_{0}^{V_{D S}} Q_{\text {total }} d V$

The drain-source current flows along the $\mathrm{X}$ direction and the device is illuminated along the $\mathrm{Y}$ direction. The gate being 
opaque, the excess carriers are generated in the extended gate depletion region and the neutral region of the channel .The optically generated electrons flow toward the drain and contributes to the drain-source current when a drain source voltage is applied. The external photovoltage developed across the junction is obtained using the relation as in [12].

$V o p=\frac{K T}{q} \ln \left(\frac{J p}{J_{S}}\right)=\frac{K T}{q} \ln \left(\frac{q v_{y} p(0)}{J_{S 1}}\right)$

Where Js is the reverse saturation current,

$\mathrm{V}_{\mathrm{y}}$ is the carrier along vertical direction perpendicular to the device surface,

$\mathrm{p}(0)$ is number of holes crossing junction at $\mathrm{y}=0$

$$
p(0)=\frac{\Pi}{4} Z\left(p_{1} Y d S^{2}+p_{2} Y d D{ }^{2}\right)
$$

Where $\mathrm{p}_{1}$ and $\mathrm{p}_{2}$ are the constants dependant on carrier lifetime under ac conditions.

$\mathrm{Y}_{\mathrm{dD}}$ and $\mathrm{Y}_{\mathrm{dS}}$ is depletion width at drain and source resp.

The calculation of photovoltage is important as it modifies the depletion width $\mathrm{Y}_{\mathrm{dG}}$ (depletion width at gate).Using abrupt junction approximation the $\mathrm{Y}_{\mathrm{dG}}$ (under dark condition) and $\mathrm{Y}^{\prime}{ }_{\mathrm{dG}}$ under illumination are calculated as given in [13].

$$
\begin{aligned}
Y_{d G}(x) & =\left(\frac{2 \varepsilon}{q N_{d r}}\left(V_{b i}+V(x)-V_{G S}\right)\right)^{1 / 2} \\
Y^{\prime}{ }_{d G}(x) & =\left(\frac{2 \varepsilon}{q N_{d r}}\left(V_{b i}+V(x)-V_{G S}-V_{o p}\right)\right)^{1 / 2}
\end{aligned}
$$

Where $\mathrm{V}(\mathrm{x})$ is channel voltage,

$\mathrm{V}_{\mathrm{bi}}$ built in potential,

Due to the photo voltage developed the effective bias across gate changes to $\left(\mathrm{V}_{\mathrm{G}}+\mathrm{V}_{\mathrm{OP}}\right)$ from $\mathrm{V}_{\mathrm{G}}$.

\section{RESULTS AND DISCUSSION}

Simulations has been carried out for multifinger N-MOSFET device with $\mathrm{Nf}=10, \mathrm{Wf}=12 \mu \mathrm{m}$ and $\mathrm{Lf}=0.36 \mu \mathrm{m}$ to study the effect illumination on a submicron device to be operated at RF.A long device with $\mathrm{W}=20 \mu \mathrm{m}$ and $\mathrm{L}=5 \mu \mathrm{m}$ is also considered for comparative analysis. The parameters used for simulation are of $0.25 \mu \mathrm{m}$ CMOS process for both the devices and is done using MATLAB. Calculations have been performed to evaluate the photo-voltage generated due to optical illumination. The drain current and transconductances have been calculated by considering the mobility effects due to vertical and lateral fields, velocity saturation, short-channel effects as channel-length modulation (CLM), charge-sharing and reverse short channel effect (RSCE) as modelled in [7] . The effect on substrate current due to impact ionization also has been accounted for. The DC characteristics are investigated with $\mathrm{V}_{\mathrm{G}}, \mathrm{V}_{\mathrm{D}}$ and $\mathrm{V}_{\mathrm{S}}$ varying from $0 \mathrm{~V}$ to $4 \mathrm{~V}$, ensuring that it covers all the regions of MOSFET operation under all varying bias conditions. The weak inversion region is specially considered as it shows significant influence of optical illumination. The transconductances are obtained from the drain current by applying appropriate conditions. The photon flux varies from $1 \times 10^{14}$ to $1 \times 10^{18}$ indicating change of optical power from $0.25 \mathrm{~mW}$ onwards.

The direct illumination of the gate results in generation of excess electron hole pairs due to absorption of radiation in depletion region. The excess carriers generated cause change in gate voltage due to photo voltage, VOP as reported in $[14,15]$.

Figure 3 indicates $I_{D}-V_{D}$ characteristics of the multifinger MOSFET for $\mathrm{V}_{\mathrm{G}}=1 \mathrm{~V}$ and $2 \mathrm{~V}, \mathrm{~V}_{\mathrm{B}}=0 \mathrm{~V}$ and varying $\mathrm{V}_{\mathrm{D}}$ under dark condition and with optical power of $0.25 \mathrm{~mW}$. It can be seen that that optical radiation increases the drain current, indicating that development of photo-voltage modulates the effective gate bias enhancing device conductivity. A similar effect is observed for higher $\mathrm{V}_{\mathrm{G}}$ also.

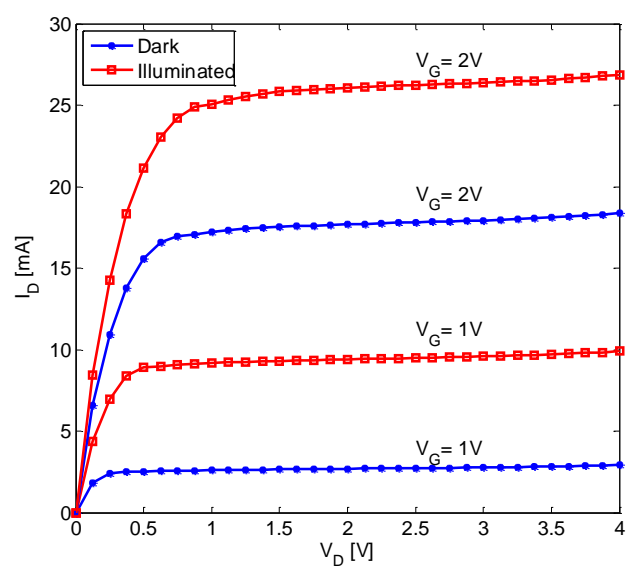

Figure 3. Drain current $V s$ drain voltage for $V_{G} 1$ Vand $2 \mathrm{~V}$, under dark and optical power of $0.25 \mathrm{~mW}$

Figure 4 indicates $I_{D}-V_{G}$ characteristics of the multifinger MOSFET for $\mathrm{V}_{\mathrm{D}}=1 \mathrm{~V}$ and $2 \mathrm{~V}, \mathrm{~V}_{\mathrm{S}}=0 \mathrm{~V}$ and varying $\mathrm{V}_{\mathrm{G}}$ under dark condition and illuminated condition with optical power of $0.25 \mathrm{~mW}$. The plot indicates that effect of optical illumination becomes more pronounced with increase in gate voltage at constant drain voltage due to channel width modulation in the MOSFET.

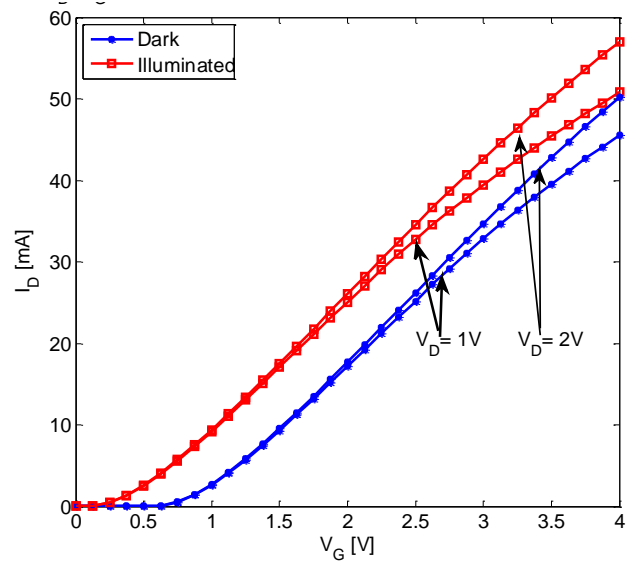

Figure 4. Drain current $V s$ gate voltage for $V_{D} 1$ Vand $2 \mathrm{~V}$,under dark and optical power of $0.25 \mathrm{~mW}$

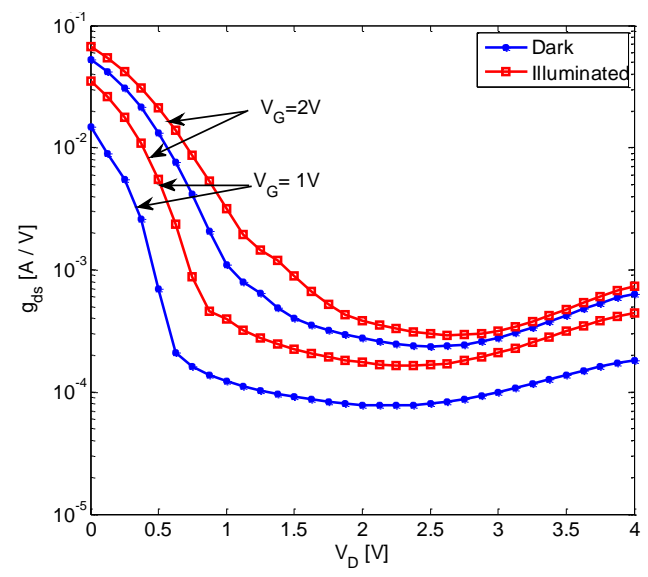

Figure 5. Drain transconductane $V s$ drain voltage for $V_{G}$ 1 Vand $2 \mathrm{~V}$, under dark and optical power of $0.25 \mathrm{~mW}$ 
Figure 5 is the plot of drain conductance under varying drain voltage with $\mathrm{V}_{\mathrm{G}}$ of $1 \mathrm{~V}$ and $2 \mathrm{~V}$. The plot indicated that with increase in drain voltage the output conductance almost remains constant in dark as well with photon flux radiation. This is due to the fact that the drain current becomes almost independent of drain voltage at higher drain voltages when device is in strong inversion. It also confirms that optical flux essentially modifies the effective gate bias rather than drain bias.

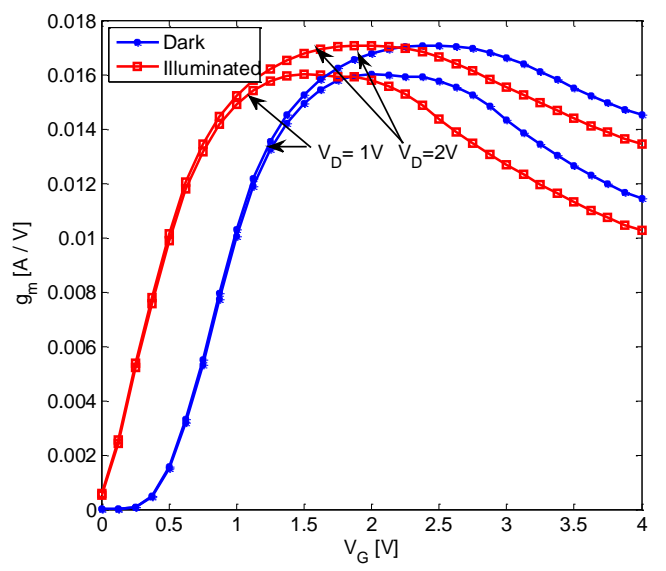

Figure 6 Gate transconductane $V s$ gate voltage for $V_{D}$ 1 Vand $2 \mathrm{~V}$, under dark and optical power of $0.25 \mathrm{~mW}$

Figure 6 are plots of gate transconductances at fixed drain voltage with varying gate voltage. The drain current is very small till the device enters inversion. Due to this the change in device gate transconductances is seen at higher gate voltage as for the characteristics as in figure 3. The transconductances curve reaches a peak and then remains almost constant. This is due to influence of $\mathrm{V}_{\mathrm{G}}$ on effective mobility. The transconductance is one of the very important factors considered in circuit design as it decides transit frequency. The optically modulated device can be used to as an additional control for the device operation.

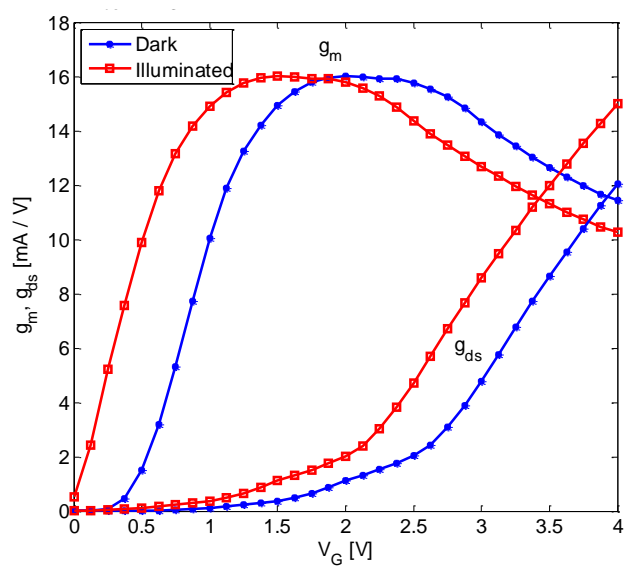

Figure 7 Gate and drain transconductane wrt Gate voltage for $\mathrm{VD} 1 \mathrm{~V}$, under dark and optical power of $0.25 \mathrm{~mW}$

Figure 7 and Figure 8 are the plots of device gate transconductances and drain conductance. Figure 7 is plot of both the parameters at varing $V_{G}$ and $V_{D}$ of $1 \mathrm{~V}$. Figure 8 is plot of both the parameters with $\mathrm{V}_{\mathrm{G}}$ of $1 \mathrm{~V}$ and varing $\mathrm{V}_{\mathrm{D}}$. These parameters are used in evaluating $\mathrm{Y}$ parameters of the device, and play a crucial role in deciding circuit gain. The circuit gain is ration of $\mathrm{gm} / \mathrm{gds}$. The influence of drain and gate source has significant impact on circuit performance and hence choice of appropriate quiescent point under optical illumination can be used to maximize circuit gain. These parameters are strongly affected by short channel effects in the device and are strongly dependant on applied bias.

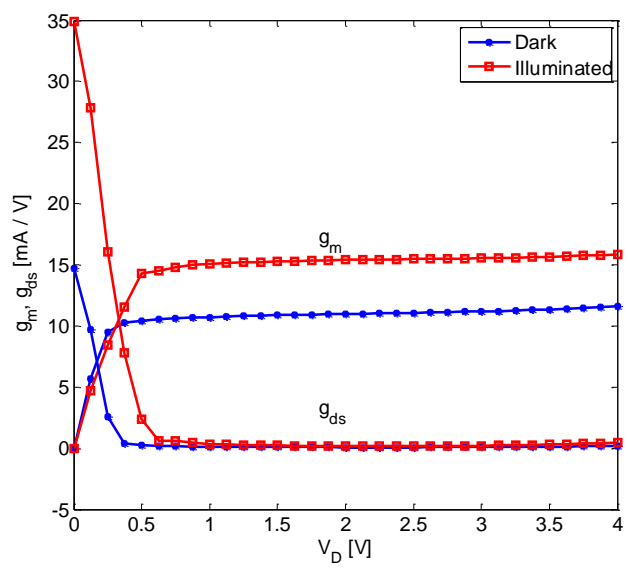

Figure 8 Gate and drain transconductane Vs drain voltage for $V_{G} 1 \mathrm{~V}$, under dark and optical power of $0.25 \mathrm{~mW}$
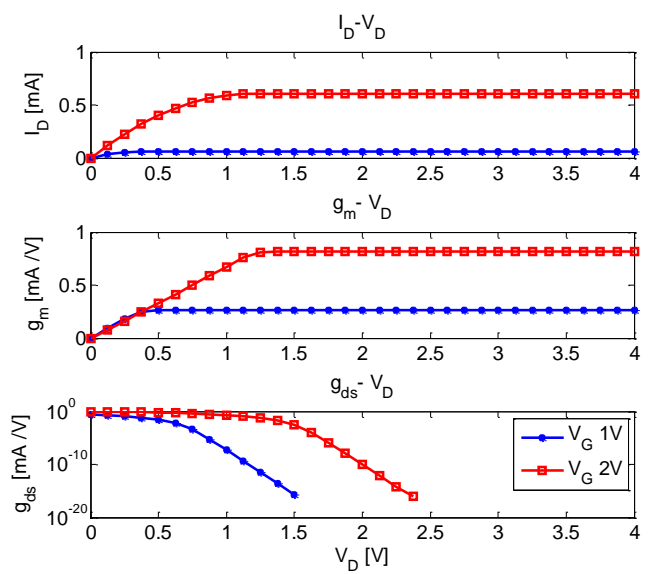

Figure 9 Drain current, gate transconductane and drain transconductane $V s$ drain voltage at $V_{G} 1 V$, under dark and optical power of $0.25 \mathrm{~mW}$ for long device.

Figure 9 is plot of drain current, gate and drain transconductance for a long channel device. The length of the device under consideration is $5 \mathrm{um}$ and width is $20 \mathrm{um}$. The plots in figure 9 can be compared with plots in figure 3,4,5. The drain characteristics are seen to be more independent of drain voltage as compared to the small dimension device. The transconductance and output conductance notably vary as compared to multifinger device. The characteristics of a long device indicate a linear relationship with the drain current. The long channel device under illumination also indicates improvement in drain current, gate transconductance and reduction in drain transconductance.

Figure 10 is plot of $\log I_{D}$ and drain voltage varying from 0 to $1 \mathrm{~V}$, for $\mathrm{V}_{\mathrm{G}}$ of $0.1 \mathrm{~V}$ and $0.2 \mathrm{~V}$. This is the weak inversion region of the MOSFET. It can be appreciated from the graph that optical radiation is significant in this region and the device characteristics can be utilized for detection purpose as the responsivity will be of enhanced in this region. Figure 11 is similar plot in weak inversion region for $\mathrm{V}_{\mathrm{G}}$ of $0.2 \mathrm{~V}$ using linear scale. This clearly indicates the device application utilizing optical radiation by selecting suitable voltage bias. 


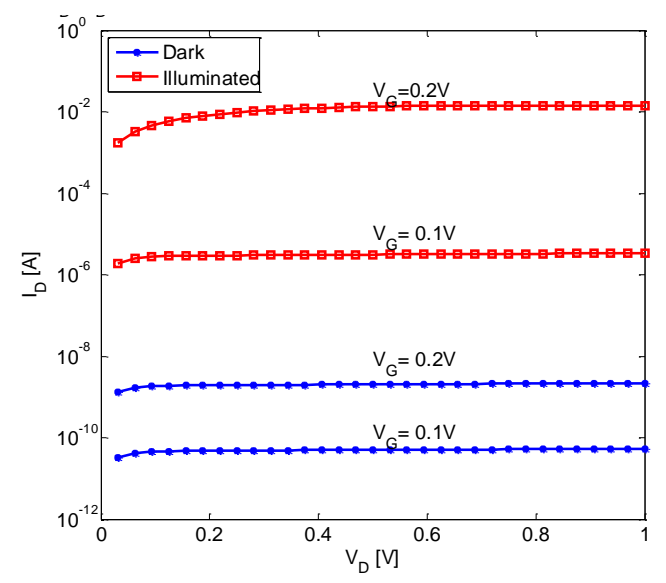

Figure 10. Drain current Vs Drain voltage for $V_{G} 0.1$ Vand $0.2 \mathrm{~V}$, under dark and optical power of $0.25 \mathrm{~mW}$

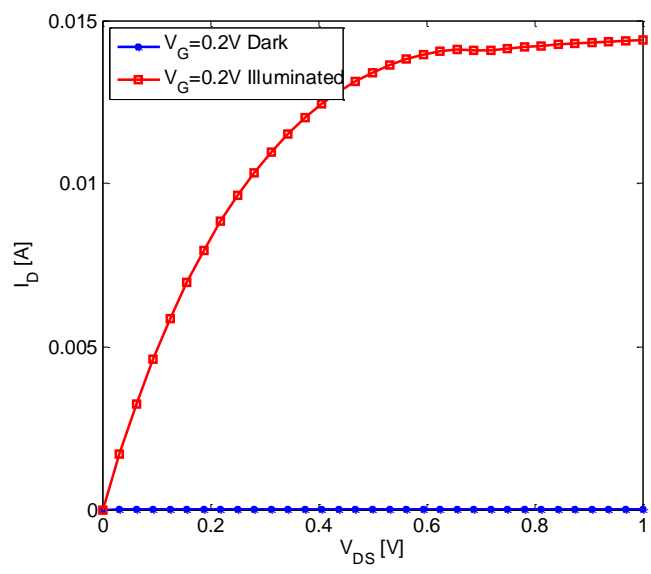

Figure 11. Drain current Vs Drain voltage for $V_{G} 0.1 \mathrm{~V}$, under dark and optical power of $0.25 \mathrm{~mW}$

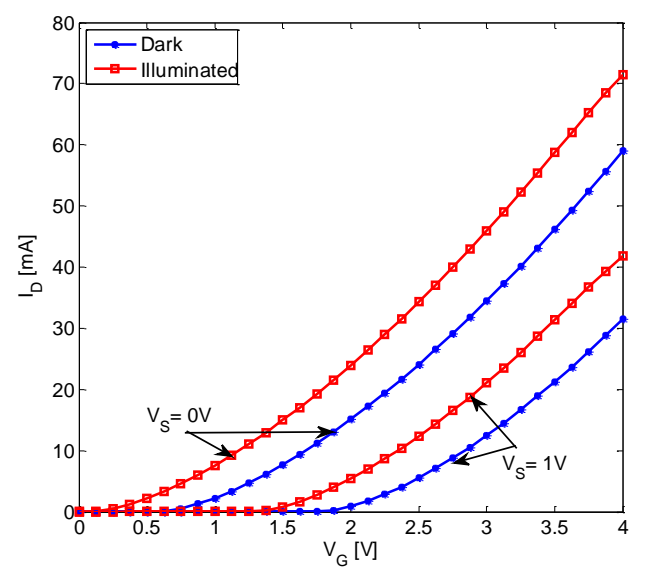

Figure 12. Drain current Vs Gate voltage for $V_{S} 0 \mathrm{~V}$ and $1 \mathrm{~V}$, under dark and optical power of $0.25 \mathrm{~mW}$

Figure 12 and Figure 13 are the plots at constant drain voltage $\mathrm{V}_{\mathrm{D}} 3 \mathrm{~V}$. Figure 12 is the plot of varying substrate bias $\mathrm{V}_{\mathrm{S}}$ of $0 \mathrm{~V}$ and $1 \mathrm{~V}$. In the conventional device the substrate bias serves as additional control gate for the device. The optical illumination on the device is seen to perform similar function of the controlling by modulating the threshold voltage of the device.

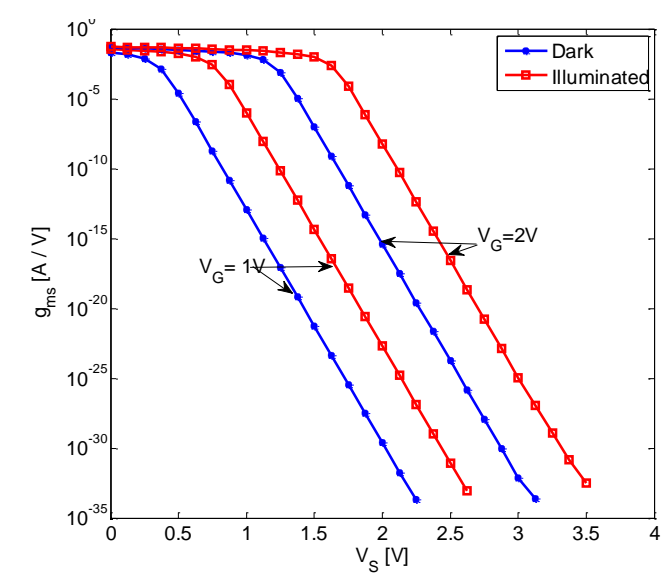

Figure 13. Source transconductance Vs Source voltage at Gate voltage $1 \mathrm{~V}$ and $2 \mathrm{~V}$, under dark and optical power of $0.25 \mathrm{~mW}$

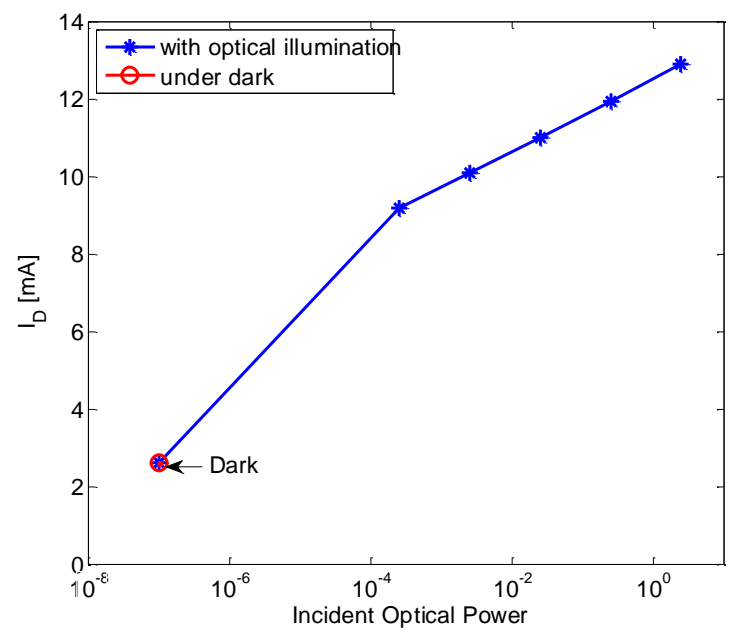

Figure 14 Drain current in dark and illuminated condition in dark and illuminated condition with photon flux of $1 x$ $10^{14}$ to $1 \times 10^{18}$

Figure 12 shows variation of drain current with varying optical power at quiescent condition with voltage $V_{G} 1 \mathrm{~V}, V_{D}$ $1 \mathrm{~V}$ and $\mathrm{V}_{\mathrm{S}}=0 \mathrm{~V}$. The drain current is seen to increase linearly with optical power. This is due to increase in channel conductivity and hence increase in inversion charge at constant bias. This enhancement of drain current explores the utility of device for detection purpose, enhance device performance and offer better control.

\section{CONCLUSIONS}

Simulation has been done for a multifinger device and a long channel device under varying bias conditions. The effects on drain current and device transconductance are observed in dark and under optical illumination. The results for the long device and multifinger device are comparable to the reported devices in dark condition. The device under optical illumination shows encouraging results for the drain current in all regions of the device operation for incident wavelength of $830 \mathrm{~nm}$. Employment of optically gated MOSFET offers an additional control port which can be useful for device operation at RF and analog circuit. Modification in composition, structure and materials will make improve application areas of the device. 


\section{REFERENCES}

[1] Daniel Foty, David Binkley, Matthias Bucher, 2002 Starting over: gm/Id based MOSFET modeling as basis for Modernized Analog design Methodolgies, , Modelling and Simulation of Microsystems 0-9708275-7-1.

[2] Singh, Jasprit,1995. Semiconductor Optoelectronics, McGraw-Hill Inc. 0-07-0S7b37-*.

[3] Fraz Sischka, Thomas Gneiting.,2001, World Scientific Publishing Company, International Journal of High Speed Electronics and Systems, Vol. 11, pp. 887-951.

[4] B. K. Mishra, Prerana N Jain, Gayatri Phade, 2010, I-V Characterization of Optically Gated MOSFET : National Conference on Electronic Technologies, 2010.

[5] Caverly Robert, 2007,CMOS RFIC Design Principles. Boston : Artech house ISBN: 10987654321.

[6] C. C. Enz, F. Krummenacher and E. A., Vittoz,1995,An anatical MOS transistor Model valid in all regions of operations and dedicated to Low-voltage and Lowcurrent applications.,Analog Integrated Circuits and System Processing Journal,Vol 8,pp 83-114.

[7] Bucher, Matthias.. Boston ,2004,. Review of the EKV3.0 MOSFET Model ,Workshop on Compact Models, NANOTECH Boston, MA

[8] Steve Hung-Min Jen, Christian C. Enz, Member, David R. Pehlke.,1999,Accurate Modeling and Parameter Extraction for MOS Transistors Valid up to $10 \mathrm{GHz}$, IEEE Transactions On Electron Devices, Vol. 46. 00189383/99.
[9] Christian., Enz, 2006,. Charge-based MOS transistor modelling : the EKV model for low-power and RF IC design ,. West Sussex , England : John Wiley \& Sons Ltd13: 978-0-470-85541-6.

[10] A.B.Bhattacharyya.,2009, Compact Mosfet Models for VLSI Designs. ,John Wiley \& Sons(Asis) Pte Ltd,,. 978$0-470-82342-2$..

[11] B.K,.Mishra, Lochan Jolly, S.C.Patil,,2011,In1-xGaxAs a next generation material for photodetectors," Cyber journal,JSAM, pp.9-16.

[12] B..K.Mishra,1995,Computer Aided modeling of solid state photodetectors, Ph.D thesis by, Birla institute of Technology, Mesra, Ranchi,

[13]Prerana Jain, B.K.Mishra, Phade G.,2012, Powergain Analysis of Optically Illuminated MOSFET, International Journal of Computer Application(09758887)-,Vol-51-No.16

[14] Prerana Jain., Mishra B.K., Gayatri Phade 2012,,'AC Performance of Optically Controlled MOSFET', Proceedings of SCEES, 2012,The IEEE website, doi : 10.1109 / SCEECS.2012.618425, ISBN:978-1-4673$1516-6$

[15] Gayatri M Phade, B.K. Mishra, Prerana Jain,' ,2012, Modeling of Optically Tailored Noise Parameters of MOSFET, International Journal of Computer Application, Proceedings on International Conference in Computational Intelligence (ICCIA2012). 